\title{
Time-Domain Homogenization of Windings in Two-dimensional Finite Element Models
}

\author{
J. Gyselinck, R. Sabariego and P. Dular
}

\begin{abstract}
In this paper the authors propose an original timedomain extension of the frequency-domain homogenization of multi-turn windings in FE models. For the winding type at hand (e.g. round conductor with hexagonal packing), an elementary $\mathrm{FE}$ model is used for determining dimensionless frequency and time domain coefficients regarding skin and proximity effect. These coefficients are readily utilized for homogenizing the winding in the FE model of the complete device. The method is successfully applied to an axisymmetric 103-turn inductor. The results agree very well with those obtained by an accurate but very expensive FE model in which all turns are finely discretised.
\end{abstract}

\section{INTRODUCTION}

For some electromagnetic devices, skin and proximity effects in the windings constitute an important design aspect. Several analytical approaches have been proposed in literature over the last decades [1], [2]. Their scope and accuracy can be increased by combining them with numerical methods, in particular the FE method [3], [4]. Most often skin and proximity effect are simply ignored in the FE resolution stage (assuming a uniform current distribution in the winding domain of the FE model) and the associated losses are estimated a-posteriori. However, in some cases the behavior of the device under study is significantly altered by the eddy-current effects and the direct inclusion of the latter in the FE equations is mandatory. In principle, this can be done by modelling each individual turn as a so-called massive conductor and by finely discretizing each of them [5]. For real-life applications, this brute-force method produces a prohibitively large number of unknowns.

The use of dedicated homogenization techniques thus seems unavoidable. Those sofar presented in literature are mainly if not entirely - limited to the frequency domain. Skin and proximity effect are accounted for by adopting a complex impedance in the electrical circuit and a complex reluctivity in the homogenized winding domain respectively. The frequency dependence of these complex quantities is obtained either analytically [3] or using an elementary FE model [6]. Round conductors with rectangular packing are mostly considered. A more general approach has recently been proposed by the authors [7]. In this paper, the latter homogenization technique is further extended to the time domain.

Manuscript received April 24, 2006. The research was partly carried out in the frame of the Inter-University Attraction Pole IAP P5/34 for fundamental research funded by the Belgian federal government.

The first author is with the Dpt. of Electrical Engineering, Université Libre de Bruxelles (ULB), Belgium (johan.gyselinck@ulb.ac.be). The second and third author are with the Dpt. of Electrical Engineering, University of Liège, Belgium. P. Dular is a Research Associate with the Belgian National Fund for Scientific Research (F.N.R.S.).

\section{Characterization OF MUlTi-TURN WindingS}

\section{A. Frequency-domain characterization}

A multi-turn winding of periodically spaced conductors of arbitrary but symmetric cross-section (e.g. round or rectangular) is considered. The equivalent radius of the conductors is is defined as $r=\sqrt{\Omega_{c} / \pi}$, where $\Omega_{c}$ is the cross-sectional surface area. The skin depth at frequency $f$ or pulsation $\omega=2 \pi f$ is given by $\delta=\sqrt{2 /\left(\sigma \omega \mu_{0}\right)}$, where $\sigma$ is the conductivity of the conductors and $\mu_{0}=4 \pi 10^{-7} \mathrm{H} / \mathrm{m}$ their permeability. The normalized or reduced frequency $X$ is defined as follows:

$$
X=\frac{r}{\delta}=\sqrt{f} \cdot r \sqrt{\pi \sigma \mu_{0}} .
$$

1) 2D FE model: A complete eddy-current effect characterization of the conductors and their packing can be carried out by means of a representative 2D FE model consisting of a central cell and one layer of cells around it [7]. In Fig. 1, such a FE model is shown for a round conductor with hexagonal packing (fill factor $\lambda$ equal to 0.7 ).

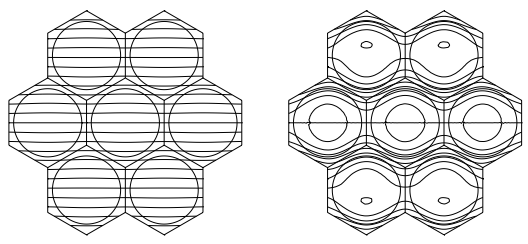

Fig. 1. Elementary FE model of winding with proximity effect flux lines at $X=1$ (left) and $X=3$ (right)

Frequency-domain FE calculations are carried out using the classical magnetic vector potential (MVP) formulation. (Complex quantities are printed in bold. $j=\sqrt{-1}$ is the imaginary unit.) The conductors are each modelled as a massive conductor and the same net sinusoidal current is imposed through them [5]. The sinusoidal induction (averaged over the central cell, horizontal and vertical component) is imposed by means of the boundary conditions. The characterization of the winding is done through the active and reactive power absorbed by the central cell [3]. By considering well chosen reference values, dimensionless coefficients are obtained, which are either equal to 1 or close to 1 for sufficiently low frequencies $(X \rightarrow 0)$.

2) Skin effect: By imposing a unit current and zero average induction, an elementary skin-effect solution at frequency $X$ is obtained. The corresponding complex impedance $Z_{\text {skin }}$ can be written as follows:

$$
\begin{aligned}
\boldsymbol{Z}_{\text {skin }}(X) & =p_{I}(X) R_{D C}+\boldsymbol{j} \omega q_{I}(X) \frac{\mu_{0} l}{8 \pi \lambda}, \\
& =R_{D C}\left(p_{I}(X)+\boldsymbol{j} q_{I}(X) \frac{X^{2}}{4 \lambda}\right)
\end{aligned}
$$


with $R_{D C}=l /\left(\sigma \Omega_{c}\right)$ the DC resistance of the conductor and $\mu_{0} l /(8 \pi)$ the internal DC inductance of a round conductor (length $l$ ). The thus defined dimensionless coefficients $p_{I}$ and $q_{I}$ depend on the winding type and the frequency $X$. As evidenced in Fig. 2, the skin-effect losses and the coefficient $p_{I}$ are practically independent of the fill factor $\lambda$.

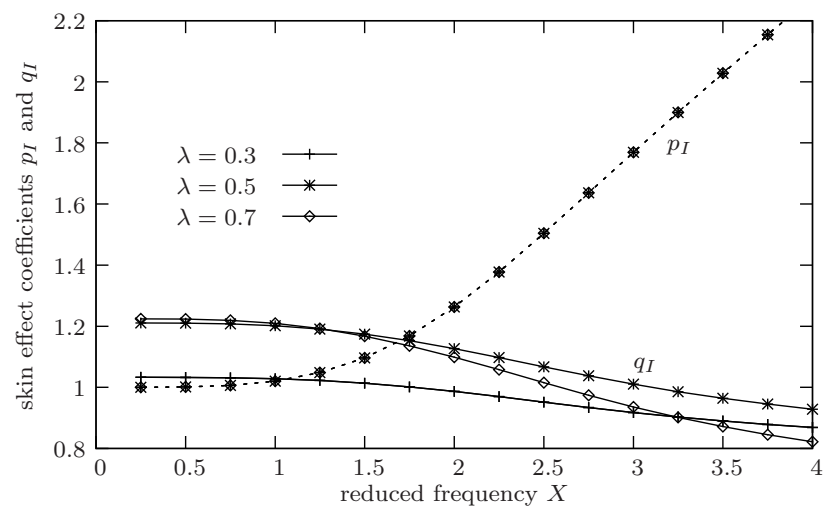

Fig. 2. Skin-effect coefficients $p_{I}$ and $q_{I}$ versus reduced frequency $X$ for different values of $\lambda$ (round conductor with hexagonal packing)

3) Proximity effect: By imposing a zero net current and a unit horizontal average induction, a pure proximity-effect flux pattern is produced in the central cell (see also Fig. 1). The corresponding complex reluctivity $\boldsymbol{\nu}_{\text {prox }}(X)$ can be written as follows:

$$
\begin{aligned}
\boldsymbol{\nu}_{\text {prox }}(X) & =q_{B}(X) \nu_{0}+\boldsymbol{j} p_{B}(X) \frac{1}{4} \lambda \sigma r^{2} \omega, \\
& =\nu_{0}\left(q_{B}(X)+\boldsymbol{j} p_{B}(X) \frac{\lambda X^{2}}{2}\right),
\end{aligned}
$$

where the factor $\lambda \sigma r^{2} \omega / 4$ in (4) follows from the analytical expression for low-frequency proximity losses in a round conductor [6]. Fig. 3 shows the dependence of the coefficients $p_{B}$ and $q_{B}$ on the frequency $X$ and the fill factor $\lambda$.

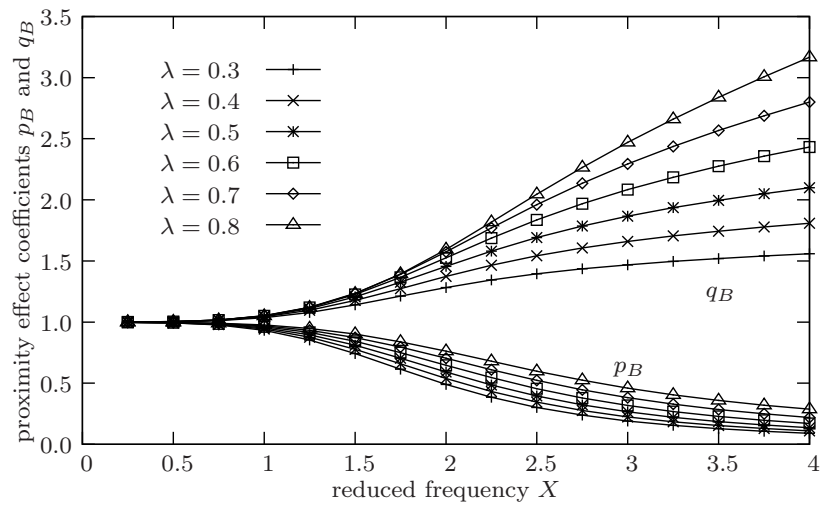

Fig. 3. Proximity effect coefficients $p_{B}$ and $q_{B}$ versus reduced frequency $X$ for different values of $\lambda$ (round conductor with hexagonal packing)

In case of an "anisotropic" winding (with e.g. conductors of rectangular cross-section), a pair of coefficients $p_{B}(X)$ and $q_{B}(X)$ has to be considered for two perpendicular directions of the flux [7].

\section{B. Time-domain extension}

1) Skin effect: A frequency-dependent complex impedance is commonly approximated by a ladder circuit comprising a finite number of lumped elements (e.g. constant resistances and inductances). This way, for the skin-effect impedance $Z_{\text {skin }}(X)$ one may obtain a approximate time-domain relation between instantaneous voltage $v(t)$ and current $i(t)$ by considering $n-1$ auxiliary currents $i_{2}(t), i_{3}(t), \ldots$ and a system of $n$ first-order differential equations in terms of the $n$ currents $[I(t)]^{T}=\left[\begin{array}{llll}i(t) & i_{2}(t) & i_{3}(t) & \ldots\end{array}\right]^{T}$ :

$$
[V(t)]=R_{D C}[I(t)]+[L] \frac{d}{d t}[I]
$$

with $[V]^{T}=\left[\begin{array}{llll}v(t) & 0 & 0 & \ldots\end{array}\right]^{T}$ and where the square matrix $[L]$ has to be determined on the basis of the given impedance $Z_{\text {skin }}(X)$. With large enough $n$, a sufficient agreement can be achieved in the relevant frequency range $\left[0, X_{\max }\right]$.

In agreement with (2-3), the system (6) is rewritten in terms of the dimensionless square matrix $\left[S^{(n)}\right]$ :

$$
[V(t)]=R_{D C}\left([I(t)]+\frac{\sigma \mu_{0} r^{2}}{8 \lambda}\left[S^{(n)}\right] \frac{d}{d t}[I]\right) .
$$

In the frequency domain, this becomes

$$
[\boldsymbol{V}]=R_{D C}\left(\left[1^{(n)}\right]+\boldsymbol{j} \frac{X^{2}}{4 \lambda}\left[S^{(n)}\right]\right)[\boldsymbol{I}],
$$

where $\left[1^{(n)}\right]$ is the $n \times n$ identity matrix. The skin-effect impedance is thus approximated as

$$
\boldsymbol{Z}_{\text {skin }}^{(n)}(X)=\frac{\boldsymbol{v}}{\boldsymbol{i}}=R_{D C}\left(\left[1^{(n)}\right]+\boldsymbol{j} \frac{X^{2}}{4 \lambda}\left[S^{(n)}\right]\right)_{(1,1)}^{-1}
$$

where the subscript $(1,1)$ indicates the first diagonal element of the inverse matrix.

In practice, a symmetrical and tridiagonal matrix $\left[S^{(n)}\right]$ allows to obtain a good agreement between $\boldsymbol{Z}_{\text {skin }}$ and $\boldsymbol{Z}_{\text {skin }}^{(n)}$. This is illustrated in Fig. 4 for the round conductor $(\lambda=0.7)$. With $n=3$ the time-domain approximation is valid beyond $X=4$.

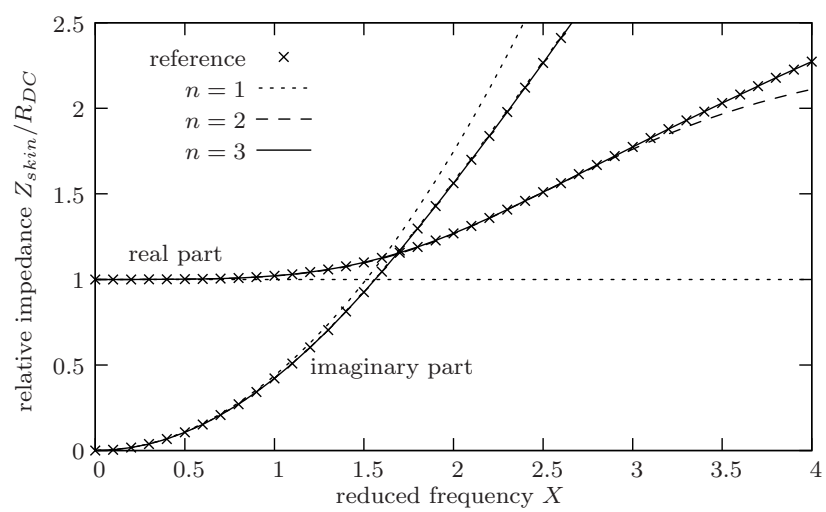

Fig. 4. Real and imaginary part of skin-effect impedance vs $X$, obtained with FE model (reference) and time-domain approximation $(n=1,2,3)$ 
2) Proximity effect: The proximity-effect reluctivity $\boldsymbol{\nu}_{\text {prox }}(X)$ gives rise to an approximate time-domain relation between instantaneous magnetic field $h(t)$ and magnetic induction $b(t)$ by considering $n-1$ auxiliary induction components $b_{2}(t), b_{3}(t), \ldots$ and a system of $n$ firstorder differential equations in terms of the $n$ inductions $[B(t)]^{T}=\left[\begin{array}{llll}b(t) & b_{2}(t) & b_{3}(t) & \ldots\end{array}\right]^{T}$. The proximity-effect counterparts of (7-9) are

$$
\begin{gathered}
{[H(t)]=\nu_{0}\left([B(t)]+\frac{\lambda \sigma \mu_{0} r^{2}}{4}\left[P^{(n)}\right] \frac{d}{d t}[B]\right),} \\
{[\boldsymbol{H}]=\nu_{0}\left(\left[1^{(n)}\right]+\boldsymbol{j} \frac{\lambda X^{2}}{2}\left[P^{(n)}\right]\right)[\boldsymbol{B}],} \\
\boldsymbol{\nu}_{\text {prox }}^{(n)}(X)=\frac{\boldsymbol{h}}{\boldsymbol{b}}=\nu_{0}\left(\left[1^{(n)}\right]+\boldsymbol{j} \frac{\lambda X^{2}}{2}\left[P^{(n)}\right]\right)_{(1,1)}^{-1},
\end{gathered}
$$

with $[H(t)]^{T}=\left[\begin{array}{lllll}h(t) & 0 & 0 & \ldots\end{array}\right]^{T}$.

With $n=3$ and the following matrix $\left[P^{(3)}\right]$

$$
\left[P^{(3)}\right]=\left[\begin{array}{ccc}
1.3880 & 0.9307 & 0 \\
0.9307 & 0.7092 & -0.1168 \\
0 & -0.1168 & 0.1916
\end{array}\right],
$$

an excellent agreement is obtained between $\boldsymbol{\nu}_{\text {prox }}(X)$ and $\boldsymbol{\nu}_{\text {prox }}^{(3)}(X)$ up to $X=4$, as can be seen in Fig. 5 .

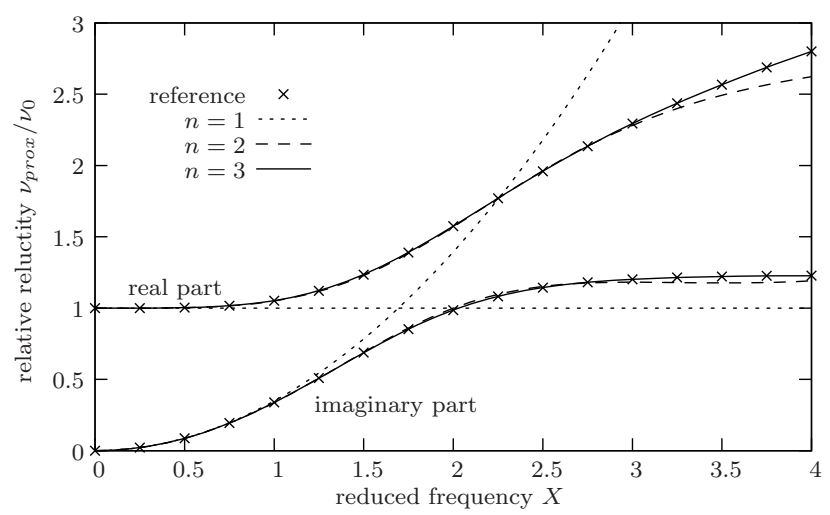

Fig. 5. Real and imaginary part of proximity-effect reluctivity vs $X$, obtained with FE model and time-domain approximation $(n=1,2,3)$

\section{Homogenization OF WINDINGS IN FE MODELS}

Adopting the 2D MVP formulation and considering the classical nodal basis functions $\alpha_{k}(x, y)$, the induction field $\vec{b}$ automatically satisfies the magnetic Gauss law. By weighing the Ampère law $\operatorname{rot} \vec{h}=\vec{j}$ with the same functions $\alpha_{l}$, a system of complex algebraic equations in terms of the nodal MVP values $\boldsymbol{a}_{k}$ results in case of a frequency-domain calculation. In the time-domain counterpart, this is a system of first-order differential equations in terms of the nodal values $a_{k}(t)$ [5].

Both in frequency and time domain homogenization, the ampère-turns of the winding are uniformly distributed over the winding domain $\Omega_{w+i}$ (conductor cross-sections plus insulation). The winding is modelled as a so-called stranded conductor, which in general is connected to other FE conductors and/or lumped electrical circuit components [5].

\section{A. Frequency domain}

Skin and proximity effect are simply taken into account by replacing $R_{D C}$ by $\boldsymbol{Z}_{\text {skin }}$ in the electrical circuit equations, and $\nu_{0}$ by $\boldsymbol{\nu}_{\text {prox }}$ in the contribution of $\Omega_{w+i}$ to the stiffness matrix of the system of FE equations [5].

\section{B. Time domain}

Skin effect is taken into account by means of the auxiliary currents $i_{2}(t), i_{3}(t), \ldots$ The associated additional electrical equations follow from (7), with fitted matrix $\left[S^{(n)}\right]$.

As for the proximity effect, additional induction components $\vec{b}_{2}(x, y, t), \vec{b}_{3}(x, y, t), \ldots$ in $\Omega_{w+i}$ are considered through auxiliary MVP components $a_{2}(x, y, t), a_{3}(x, y, t), \ldots$ For these components, the same basis functions $\alpha_{k}(x, y)$ are adopted and the nodal values $a_{2, k}(t), a_{3, k}(t), \ldots$ associated with nodes $k$ situated in $\Omega_{w+i}$ or on its boundary constitute additional degrees of freedom.

Weakly imposing the Ampère law and the additional equations (10), the resulting system of differential equations in terms of all nodal MVP values $a_{k}(t), a_{2, k}(t), a_{3, k}(t), \ldots$ (constituting vector $[A]$ ) can be written as follows

$$
[K][A]+[M] \frac{d}{d t}[A]=[J],
$$

where the entries of stiffness matrix $[K]$ and righthand side vector $[J]$ have the classical expression [5]: namely the integral over the complete FE domain (or subdomain $\Omega_{w+i}$ ) of $\nu_{0} \operatorname{grad} \alpha_{k} \cdot \operatorname{grad} \alpha_{l}$ and integral of the uniform current density multiplied by $\alpha_{k}$.

The different MVP components are solely coupled through the symmetric and positive definite mass matrix $[M]$. Following (10), its entries read

$$
\frac{\lambda \sigma r^{2}}{4} P_{i, j}^{(n)} \int_{\Omega_{w+i}} \operatorname{grad} \alpha_{k} \cdot \operatorname{grad} \alpha_{l} d \Omega,
$$

where for sake of brevity the type of symmetry (i.e. translational or axial) has not been considered explicitly [5]. The total

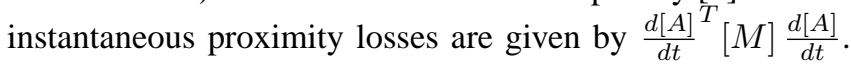

\section{Application example}

The 2D FE model of an axisymmetric 103-turn inductor having a magnetic core (relative permeability 1000) is shown in Fig. 6. The conductor has a round cross-section $(r=$ $\left.0.52 \mathrm{~mm}, \sigma=6 \cdot 10^{7} \mathrm{~S} / \mathrm{m}, \lambda=0.7\right)$. The fringing flux due to the $1 \mathrm{~mm}$ airgap in the core effects a significant increase of the proximity losses. For illustrating this, two different 103-turn windings are considered: the one shown in Fig. 6 (referred to as "winding 1", $R_{D C}=0.190 \Omega$ ), and the one whose crosssection is moved $1 \mathrm{~mm}$ to the right, away from the airgap (referred to as "winding 2", $R_{D C}=0.203 \Omega$ ).

\section{A. Frequency-domain results}

Frequency-domain calculations with imposed unit current are carried out, with the reduced frequency $X$ ranging from 0.1 up to 4 ( $X$ equal to $1,2,3$ and 4 corresponds to $f$ equal to 


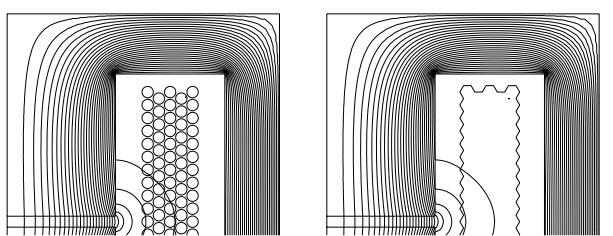

Fig. 6. 2D FE model of axisymmetric inductor: flux lines at $X=1$ obtained with fine model (left) and with homogenized model (right)

$15.58,62.34,140.3$ and $249.4 \mathrm{kHz}$ ). With a fine model (characteristic element size equal to $r / 9$ in the conductor crosssections, 45000 complex unknowns in total), one calculation takes 50 to $129 \mathrm{~s}$ (increasing with $X$ ) on a Pentium 4, $2 \mathrm{GHz}$. With a homogenized model (7000 complex unknowns) this is between 0.9 and $1.5 \mathrm{~s}$. Some flux patterns are shown in Fig. 7 (winding 1).

The winding resistance and inductance obtained with the fine model and the homogenized model $(n=3)$ are compared in Fig. 8. Up to say $X=2$ the agreement is excellent. At higher frequencies a slight deviation appears. Given the low DC resistance and the skin-effect factor $p_{I}$ shown in Fig. 2, the joule losses in the winding are nearly entirely due to the proximity effect (say for $X>1$ ). Clearly, winding 1 is more subjected to the fringing field than winding 2 .
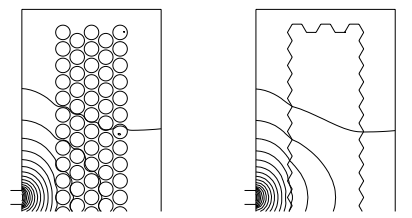

Fig. 7. Flux lines in winding window at $X=3$, obtained with fine mode (left) and homogenized model (right)

\section{B. Time-domain results}

Next time-stepping simulations with imposed triangular current of fundamental frequency $X=2$ (fundamental period $T=1 / f=16.04 \mu \mathrm{s}$ ) are carried out. Only half a period is simulated, with time step $\Delta t=T / 200$, during which the current goes from 0 to $1 \mathrm{~A}$, and back to 0 . Fig. 9 shows how the total joule losses vary in time. The homogenization method (with $n=3$ ) produces again accurate results.

\section{CONCLUSIONS}

The frequency and time domain homogenization methods presented in this paper allow to take into account the skin and the proximity effect in a winding in a $2 \mathrm{D}$ FE model with good accuracy and at a reasonable computational cost. To this end, the winding type is first characterized by means of a computationally cheap 2D FE model, leading to four dimensionless but frequency-dependent coefficients. This frequencydomain characterization is next extended to the time domain, requiring the introduction of a limited number of additional unknowns for the homogenized winding. This method can be straightforwardly extended to 3D FE models.
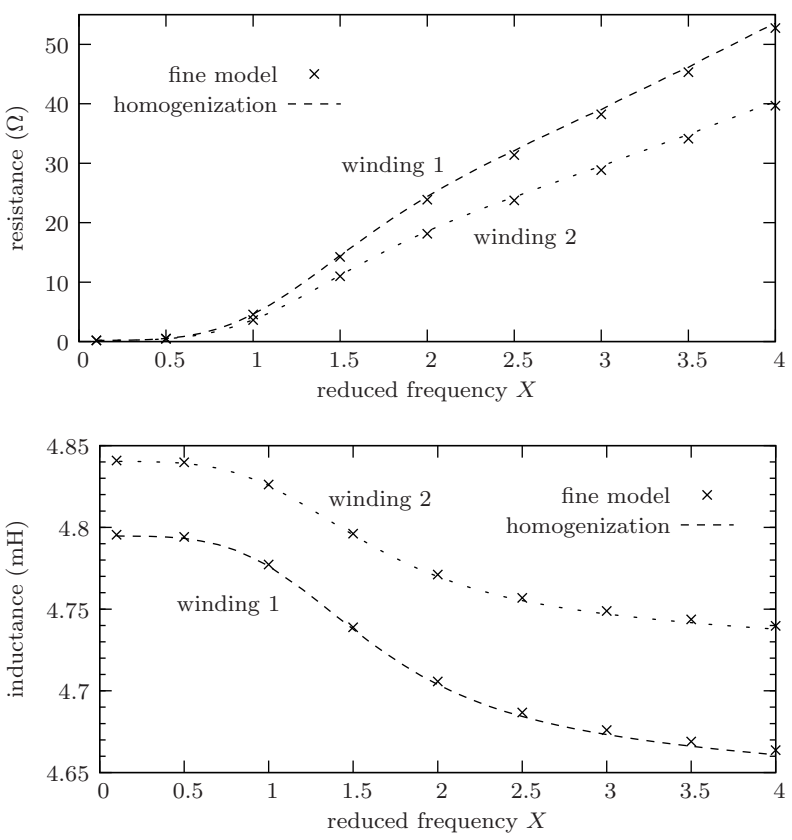

Fig. 8. Resistance (up) and inductance (below) as a function of reduced frequency $X$ obtained with fine model and with homogenization $(n=3)$

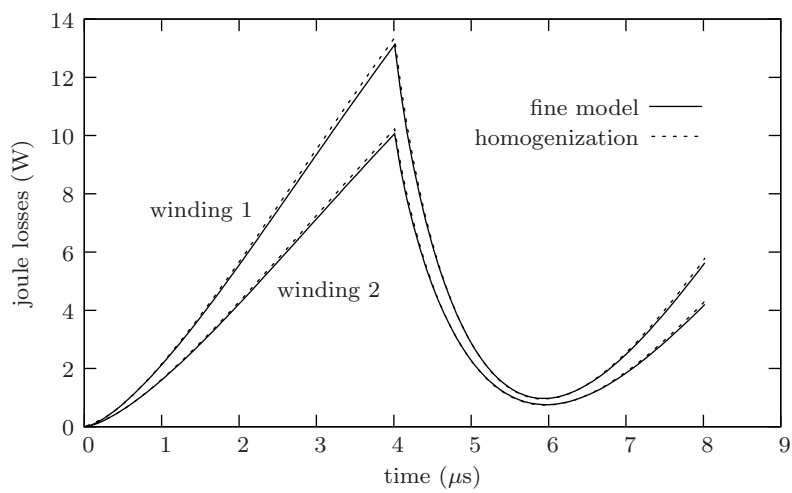

Fig. 9. Joule losses versus time obtained with fine model and homogenized model $(n=3)$

\section{REFERENCES}

[1] P. Dowel, "Effects of eddy currents in transformer windings", Proc. IEEE, vol. 113, pp. 1387-1394, August 1966.

[2] F. Ferreira, "Improved analytical modeling of conductive losses in magnetic components", IEEE Trans. Power Electron., vol. 9, no. 1, pp. 127-131, January 1994.

[3] O. Moreau, L. Popiel and J. L. Pages, "Proximity losses computation with a 2D complex permeability modelling", IEEE Trans. on Magn., vol. 34, no. 5, pp. 3616-3619, September 1998.

[4] C. Sullivan, "Computationally efficient winding loss calculation with multiple windings, arbitrary waveforms, and two-dimensional or threedimensional field geometry", IEEE Trans. on Power Electron., vol. 16, no. 1, pp. 142-150, January 2001.

[5] P. Lombard, G. Meunier, "A general purpose method for electric and magnetic combined problems for $2 \mathrm{D}$, axisymmetric and transient systems", IEEE Trans. on Magn., vol. 29, no. 2, pp. 1737-1740, March 1993.

[6] A. Podoltsev and B. Lebedev, "Analysis of effective resistance and eddy-current losses in multiturn winding of high-frequency magnetic components", IEEE Trans. on Magn., vol. 39, no. 1, pp. 539-548, January 2003.

[7] J. Gyselinck, P. Dular, "Frequency-domain homogenization of bundles of wires in 2D magnetodynamic FE calculations", IEEE Trans. on Magn., vol. 41, no. 1, pp. 1416-1419, April 2005. 